\title{
Determination and quantification of arbutin in plants using stable isotope dilution liquid chromatography-mass spectrometry
}

\author{
Tae Jin Kim ${ }^{1}$ - Young Jin Park ${ }^{1}$ - Sang Un Park ${ }^{2}$. \\ Sun-Hwa $\mathrm{Ha}^{3} \cdot$ Jae Kwang Kim $^{1}$ (D)
}

Received: 4 June 2018/Accepted: 15 July 2018/Published online: 23 July 2018

(C) The Korean Society for Applied Biological Chemistry 2018

\begin{abstract}
Arbutin is a very safe whitening agent for human skin. Since it is more expensive than other agents and has a challenging synthesis, novel methods to obtain this valuable agent are needed. In this study, we developed a precise and accurate method to detect and quantify arbutin using stable isotope dilution liquid chromatography-mass spectrometry (LC-MS). One challenge that needed to be overcome was the matrix effect occurring during the LCMS analysis due to the analyte ionisation enhancement or suppression in the electrospray ionisation source by coeluting compounds. Notably, arbutin had different matrix effects in the various sample matrices. A solution to this problem was the use of $\left[d_{4}\right]$-arbutin as a stable isotopelabelled internal standard (SIL-IS), as it compensated the matrix effect of arbutin because it was affected by almost the same matrix effect. The validation of the developed method showed excellent linearity $\left(r^{2}=1.000\right)$, precision
\end{abstract}

Electronic supplementary material The online version of this article (https://doi.org/10.1007/s13765-018-0385-1) contains supplementary material, which is available to authorized users.

Tae Jin Kim and Young Jin Park are co-first authors who contributed equally to this work.

Jae Kwang Kim

kjkpj@inu.ac.kr

1 Division of Life Sciences, College of Life Sciences and Bioengineering, Incheon National University, Incheon 22012, Republic of Korea

2 Department of Crop Science, Chungnam National University, 99, Daehak-Ro, Yuseong-gu, Daejeon 34134, Republic of Korea

3 Department of Genetic Engineering and Graduate School of Biotechnology, Kyung Hee University, Yongin 17104, Republic of Korea (relative standard deviation $\leq 2.5 \%$ ), accuracy (recovery, 97.42-98.52\%), limit of detection $(0.03 \mu \mathrm{g} / \mathrm{mL})$, and limit of quantification $(0.1 \mu \mathrm{g} / \mathrm{mL})$. Finally, the method of arbutin detection was applied to blueberry leaves to compare the precision and accuracy results obtained by performing stable isotope dilution using LC-MS and gas chromatography-mass spectrometry. The method was applied to strawberry leaves and pear peels, indicating that the SIL-IS method can be expected to find application in the arbutin analysis in other plants.

Keywords Arbutin · Gas chromatography-mass spectrometry $\cdot$ Liquid chromatography-mass spectrometry - Matrix effect . Plant - Stable isotopelabelled arbutin

\section{Introduction}

Arbutin, a naturally occurring glycoside of hydroquinone, acts as an inhibitor of the enzyme tyrosinase, which converts tyrosine into melanin in the skin $[1,2]$. For this reason, arbutin is widely used in cosmetics as the whitening agent for human skin. However, considering the fact that this chemical has reportedly exhibited hazardous effects such as nephrotoxic, hepatotoxic, carcinogenic, and mutagenic properties when tested on animals, it should be used with caution [3]. In addition, arbutin is more expensive than other whitening agents, such as ascorbic acid, hydroquinone, kojic acid, $\beta$-carotene, and $\alpha$-tocopherol, and is difficult to synthesise. Therefore, in order to investigate novel methods to obtain this valuable agent, it is crucial to screen new potential natural plant resources. 
Previous studies have employed analytical methods such as thin layer chromatography [4], micellar liquid chromatography [5], capillary electrophoresis [6, 7], high-performance liquid chromatography (HPLC) [8-10], liquid chromatography-mass spectrometry (LC-MS) [11, 12], and gas chromatography-mass spectrometry (GC-MS) [13-15] for screening and determining arbutin. Recently, mass spectrometry-based approaches have been increasingly employed for the determination of arbutin due to their high selectivity and sensitivity. However, the GC-MS method requires an additional step of derivatisation, i.e. by trimethylsilylation, which reduces the polarity of the functional groups of arbutin and makes it volatile [16]. In addition, the compounds resulting from the derivatisation step are unstable over time and include multiple TMS derivatives such as 5TMS and 4TMS arbutin derivatives [17]. To overcome these problems, previous studies employed the stable isotope dilution method with $\left[d_{4}\right]$-arbutin as an internal standard (IS), which successfully eliminates the issues of multiple TMS derivatives with low stability [17].

Another method with high selectivity and sensitivity is the LC-MS analysis. However, this approach also has problems such as the matrix effect, which is the enhancement or suppression of the analyte ionisation in the electrospray ionisation (ESI) source by co-eluting compounds [18-20], thereby making the data produced from the LCMS less reliable. Importantly, since GC-MS generally employs electron impact ionisation, which is hard ionisation, it is not affected by the matrix effect due to the sufficient ionisation energy. In contrast, LC-MS uses ESI, which is soft ionisation, thereby inducing the matrix effect. For this reason, researchers have focused on developing various approaches to overcome this issue. [21]. These include approaches such as post-column standard infusion [22], echo-peak technique [23, 24], isotope dilution $[25,26]$, and internal standard usage [27]. However, some of these techniques have certain disadvantages. For example, the post-column standard infusion does not provide a quantitative level by the matrix effect for targeting analytes [22]. Also, in the echo-peak technique, the ionisation efficiency which occurs from the matrix effect might be different even if the echo-peak elutes close to the target analyte [23, 24]. In addition, the internal standard usage approach applies structural analogues as ISs, which might have different retention times and be affected by different matrix effects than the analyte. Nevertheless, these issues could be solved by the isotope dilution method with the use of a stable isotope-labelled IS (SIL-IS), which represents a derivative of the target analyte in which several atoms are replaced by stable isotopes. Since the SIL-IS and analyte of interest have almost the same chemical structures and properties, they would co-elute and be affected by almost the same matrix effects. In addition, other bioactive compounds such as niacin, $\gamma$-aminobutyric acid, and betaine have already been analysed using the stable isotope dilution method, which allowed for several challenges such as the matrix effect, TMS derivatives, and optimisation for rapid quantification to be solved [28-30]. However, problems such as cross-contamination and crosstalk in the ESI source should be carefully addressed and demonstrated through method validations [20].

Until now, the detection of arbutin using the LC-MS method combined with an isotope dilution has not been reported. Therefore, in this study, we developed a novel isotope dilution LC-MS method for arbutin quantification using $\left[d_{4}\right]$-arbutin as an IS. Moreover, we analysed the matrix effect using two kinds of blueberry leaves, strawberry leaves, and pear peels. Finally, we compared this novel isotope dilution LC-MS method to the previously reported isotope dilution GC-MS method which employs the same SIL-IS [17].

\section{Materials and methods}

\section{Chemicals and samples}

Highbush blueberry cultivars (Vaccinium corymbosum L. cv. Brigitta and Duke) and strawberries (Fragaria spp.) were cultivated at the experimental farm of the Incheon National University (Incheon, Korea), while pears (Pyrus pyrifolia) were purchased from the market. Blueberry leaves and pear peels were freeze-dried and then ground using a mortar and pestle. Strawberry leaves were ground in liquid nitrogen. The resulting samples were stored at $-80{ }^{\circ} \mathrm{C}$ before extraction. Additional compounds used in this study included $\left[d_{4}\right]$-arbutin (TLC Pharmaceutical Standards Ltd, Ontario, Canada), arbutin ( $\geq 98 \%$, SigmaAldrich, St. Louis, MO, USA), salicin ( $\geq 99 \%$, SigmaAldrich, St. Louis, MO, USA), N,O-bis(trimethylsilyl)trifluoroacetamide with trimethylchlorosilane (BSTFA containing 1\% TMCS, Sigma-Aldrich, St. Louis, MO, USA), formic acid (99\%, Wako Pure Chemicals Co. Ltd., Osaka, Japan), and acetonitrile (Honeywell Burdick and Jackson, NJ, USA). Tertiary distilled water was produced using a Millipore water purification system (Milli-Q Direct 8, Millipore, Molsheim, France).

\section{Sample preparation and extraction}

The powdered samples $(10 \mathrm{mg})$ were added to methanol $(1 \mathrm{~mL})$ solutions of the internal standards $\left(\left[d_{4}\right]\right.$-arbutin or salicin, $1 \mu \mathrm{g} / \mathrm{mL}$ ), and the resulting mixtures were subjected to sonication for $30 \mathrm{~min}$ (1510R-DTH ultrasonic cleaner, Branson, Danbury, US). Then, each sample was 
centrifuged at $16,000 \times \mathrm{g}$ and $5{ }^{\circ} \mathrm{C}$ for $3 \mathrm{~min}$ (Tomy MX307, Tomy, Tokyo, Japan). The supernatant was collected in a fresh tube, and the methanol fraction was subsequently evaporated using a centrifugal concentrator (CC-105, Tomy). When the GC-MS method was used, BSTFA $(100 \mu \mathrm{L})$ was added to the resulting solution for the derivatisation step, and the mixture was incubated for $30 \mathrm{~min}$ at $60{ }^{\circ} \mathrm{C}$ and $1200 \mathrm{rpm}$ using Thermomixer Comfort (model 5355, Eppendorf AG, Hamburg, Germany). When the LC-MS method was employed instead, the dried samples were re-dissolved in tertiary distilled water $(1 \mathrm{~mL})$, filtered through a syringe filter $(0.5 \mu \mathrm{m})$, and centrifuged $\left(16,000 \times \mathrm{g}, 5^{\circ} \mathrm{C}, 3 \mathrm{~min}\right)$, thereby skipping the derivatisation step.

\section{Matrix effect of arbutin}

The matrix effect represents the analyte ionisation efficiency in the sample matrix. In this study, the respective matrix effects in blueberry leaves, strawberry leaves, and pear peels were determined. As described above, $1.0 \mu \mathrm{g} /$ $\mathrm{mL}$ solutions of arbutin, $\left[d_{4}\right]$-arbutin, salicin standard solution, extracts of samples, and extracts of samples spiked with $1.0 \mu \mathrm{g}$ arbutin were prepared. The resulting samples were analysed using LC-MS $(n=10)$. The percentage of matrix effect was calculated as follows: $\% \mathrm{ME}=100 \times\left(\right.$ analyte area $a_{\text {post extraction spiked matrix }-}$ analyte area $\left.a_{\text {post extraction matrix }}\right) /$ analyte area $a_{\text {standard }}$. A matrix effect value of $100 \%$ indicates no effect, while values above and below $100 \%$ display an ionisation enhancement and suppression, respectively. Moreover, the matrix effect correction factor was calculated as follows: $\% \mathrm{ME}_{\text {Arbutin/ }}$ $\% \mathrm{ME}_{\text {Internal standard }}$, where a matrix effect correction factor of 1.0 indicates that arbutin and the IS were affected by the same matrix effect.

\section{Method validation for the arbutin assay}

Nine calibration points with different quantities of arbutin (equivalent from 0.02 to $5.00 \mu \mathrm{g} / \mathrm{mL}$ ) and a fixed amount of IS $(1.00 \mu \mathrm{g})$ were prepared. The nine samples were analysed on the same day using LC-MS to determine the precision, accuracy, and linearity of the method. Then, quantitative calculations were done based on the corrected peak area ratios of arbutin and the IS. The precision was indicated as a percentage of the relative standard deviation (RSD\%), while the accuracy was measured as a percentage of the recovery (recovery \%). Both the precision and accuracy were determined from the calibration level. In order to evaluate the precision and accuracy of the method, three repetitions of the standard were performed using three different concentrations $(0.63,1.25$, and $5.00 \mu \mathrm{g} /$
$\mathrm{mL}$ ). The limit of detection (LOD) and limit of quantification (LOQ) were calculated by $3.3 \alpha / \beta$ and $10 \alpha / \beta$, respectively, where $\alpha$ is the standard deviation of the $y$ intercept of the calibration curve and $\beta$ is the slope of the calibration curve. Method validation was performed according to appendix $\mathrm{F}$ of the AOAC guidelines [31]. The accuracy and precision of the LC-MS and GC-MS assays in the sample matrix were determined by analysing the blueberry leaves samples and the ones spiked with known concentrations of arbutin stock solution $(n=3,0.10$, and $0.20 \mu \mathrm{g}$ ) during the pre-extraction. In addition, the assays were performed intra- and inter-day (intra-day: examined within 1 day, inter-day: examined daily for 3 days).

\section{LC-MS analysis}

The determination of arbutin was performed on an Agilent 1260 Infinity HPLC (Agilent technologies, Santa Clara, CA, USA), which was equipped with an auto sampler, vacuum degasser, variable wavelength detector, and Agilent HPLC workstation. Arbutin was separated using Develosil ODS-UG-5 column $(2.0 \times 250 \mathrm{~mm}$, Nomura Chemical, Seto, Japan) at $40{ }^{\circ} \mathrm{C}$. The mobile phase was acetonitrile with $0.1 \%$ formic acid (solvent A) and tertiary distilled water with $0.1 \%$ formic acid (solvent B). The flow rate was $0.3 \mathrm{~mL} / \mathrm{min}$, and the injection volume was $5 \mu \mathrm{L}$. The gradient conditions were as follows: $0 \mathrm{~min}, 2 \% \mathrm{~A}: 98 \%$ B; 5 min, $2 \%$ A:98\% B; 10 min, $10 \%$ A:90\% B; 15 min, $20 \%$ A: $80 \%$ B; $20 \mathrm{~min}, 20 \%$ A: $80 \%$ B; $30 \mathrm{~min}, 75 \%$ A:25\% B; $35 \min , 75 \%$ A:25\% B; 40 min, $2 \%$ A:98\% B. MS analysis was performed on an Agilent 6120 Quadrupole LC-MS equipped with an electrospray ionisation/mass spectrometry (ESI/MS) and running on an Open LAB CDS ChemStation Edition Rev. C.01.07 software (Agilent Technologies). The ESI/MS conditions were optimised as follows: positive ion mode; drying gas $\left(\mathrm{N}_{2}\right)$, $12 \mathrm{~L} / \mathrm{min}$; nebuliser pressure, $35 \mathrm{psig}$; drying gas temperature, $350{ }^{\circ} \mathrm{C}$; capillary voltage, $3000 \mathrm{~V}$; fragmentor voltage, $110 \mathrm{~V}$; selected ion monitoring (SIM) mode, $[\mathrm{M}+\mathrm{Na}]^{+} \mathrm{m} / z, 295.1$ (arbutin), $[\mathrm{M}+\mathrm{Na}]^{+} \mathrm{m} / z, 299.1$ ([ $\left.d_{4}\right]$-arbutin), and $[\mathrm{M}+\mathrm{Na}]^{+} \mathrm{m} / 2309.1$ (salicin).

\section{GC-MS analysis}

GC-MS was performed using a Rtx-5MS column (30 m length, $0.25 \mathrm{~mm}$ diameter, $0.25 \mu \mathrm{m}$ thickness, Restek, Bellefonte, PA, USA). GCMS-QP2010 Ultra System (Shimadzu, Kyoto, Japan) was equipped with an autosampler (AOC-20i, Shimadzu) and running on a Labsolutions GCMSsolution software version 4.11 (Shimadzu). The samples $(1.0 \mu \mathrm{L}$ each) were injected with a 10:1 split ratio, and the temperatures of injection, ion source, and interface were 250,230 , and $280{ }^{\circ} \mathrm{C}$, 
Fig. 1 Chromatograms and mass spectra of arbutin and $\left[d_{4}\right]$ arbutin. (A) Selected ion chromatograms of arbutin $(\mathrm{m} / \mathrm{z}$ 295.1) and $\left[d_{4}\right]$-arbutin $(\mathrm{m} / \mathrm{z}$ 299.1). Mass spectra of (B) arbutin and (C) $\left[d_{4}\right]$-arbutin

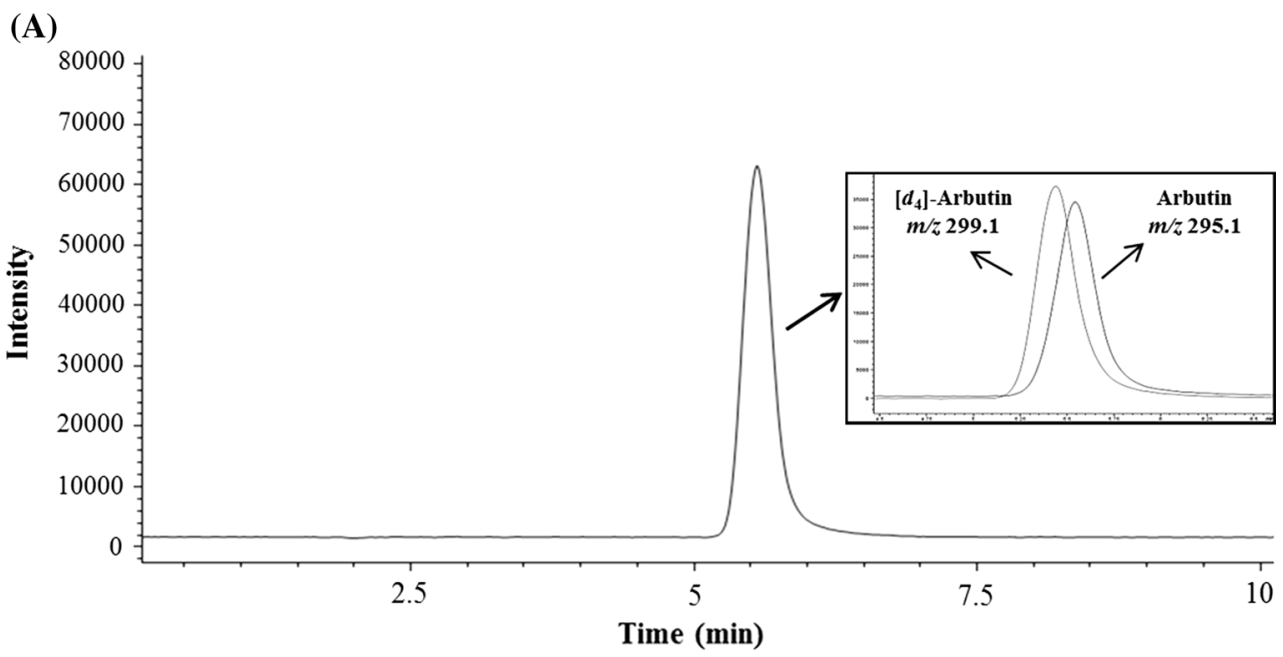

(B)

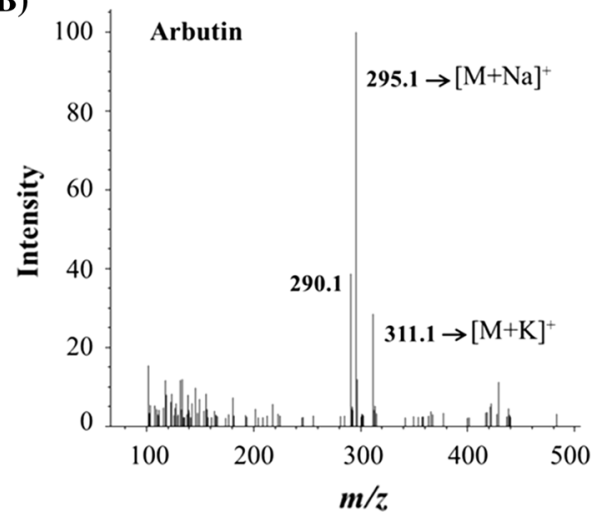

(C)

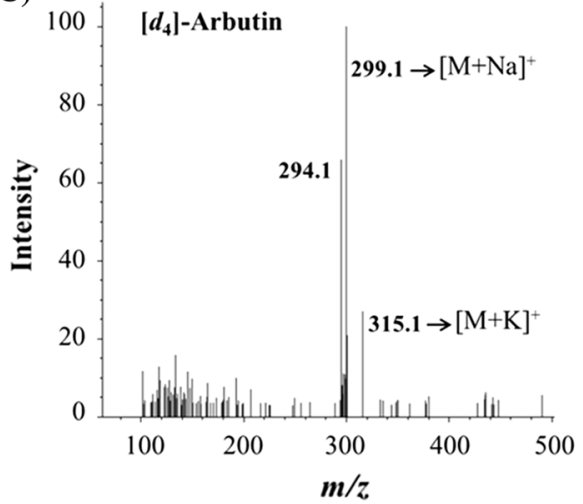

respectively. Helium was used as the carrier gas, and the column flow rate was set to $1.0 \mathrm{~mL} / \mathrm{min}$. The oven temperature was kept for $2 \mathrm{~min}$ at an isothermal heating of $200{ }^{\circ} \mathrm{C}$, after which it was raised to $275{ }^{\circ} \mathrm{C}$ at a rate of $6{ }^{\circ} \mathrm{C} / \mathrm{min}$ and maintained at that temperature for $2 \mathrm{~min}$. The MS detector was in an electron ionisation mode with a selected ion monitoring mode at $\mathrm{m} / z, 254$ (arbutin), $\mathrm{m} / \mathrm{z}, 258$ ([ $\left.d_{4}\right]$-arbutin), and $\mathrm{m} / \mathrm{z} 361$ (salicin). The mass spectra were generated during the runtime range of 2.00-16.50 min. All analytes were identified according to the retention times, and the respective mass spectra were confirmed by the standards.

\section{Results and discussion}

\section{Matrix effect}

The matrix effect is caused by the co-elution of compounds from the matrix which affects the ionisation efficiency and reproducibility of the ionisation source towards the target analytes. Since each sample has a different matrix effect and the data from the LC-MS analysis influenced by the respective matrix effect are less reliable, it is necessary to develop an analytical method which would limit the matrix effects of various samples [32]. In an effort to overcome this problem, we examined the matrix effects in blueberry and strawberry leaves, as well as pear peels, which are all known to contain arbutin [11, 33, 34]. Each sample was analysed in 10 replicates. The matrix effects of arbutin in the samples were found to be in the range of $53-78 \%$, which indicated an ionisation suppression (Table S1).

Lamien-Meda et al. [14] reported on the use of salicin as an IS in the GC-MS analysis of arbutin. In addition, another study presented a stable isotope dilution GC-MS analysis method using $\left[d_{4}\right]$-arbutin as an IS to overcome any problems connected to the stability of arbutin TMS derivatives [17]. The results from this method were then compared with the ones obtained when salicin was used as an IS. Therefore, in order to address the ionisation suppression, we compared the LC-MS assays resulting when salicin was used as an analogue IS and $\left[d_{4}\right]$-arbutin as an SIL-IS. When $\left[d_{4}\right]$-arbutin was employed as an IS, all analytes were detected within 6 min (Fig. 1A). The 


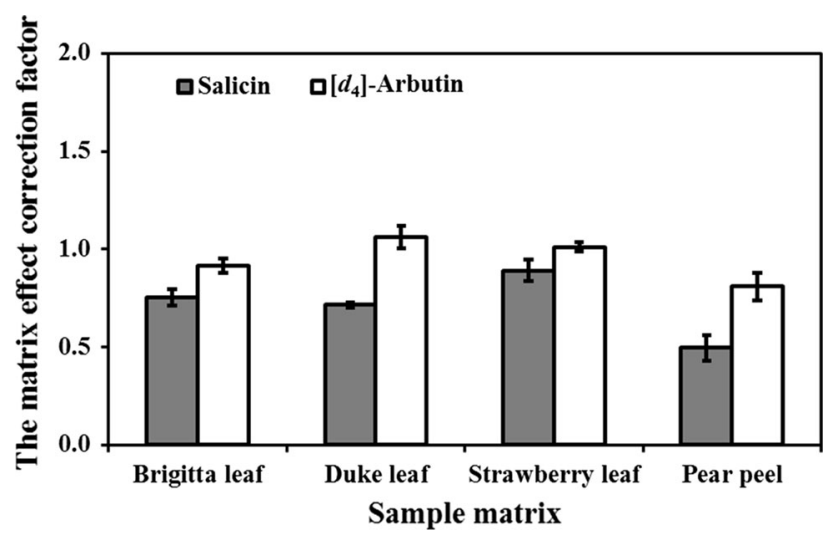

Fig. 2 Matrix effect correction factors from the sample matrices and the internal standards. Brigitta and Duke leaves represent highbush blueberry cultivars (Vaccinium corymbosum L.) $(n=10)$

analysis of the LC-MS mass spectra was performed in positive mode (Fig. 1B, C), and since the molecular ion $[\mathrm{M}+\mathrm{Na}]^{+}$had the highest intensity (arbutin: $\mathrm{m} / z, 295.1$, [ $\left.d_{4}\right]$-arbutin: $m / z$ 299.1, and salicin: $m / z$ 309.1), it was selected as a quantification ion. When salicin was used as an analogue IS, the \% MEs for arbutin and salicin were in the ranges of $49.57-92.66$ and $80.89-103.52 \%$, respectively (Table S1). Moreover, the \% ME values for arbutin and salicin differed for the different sample matrices. Figure 2 shows a bar graph displaying the matrix effect correction factors under various sample matrix conditions. As can be observed, the matrix effect correction factors obtained when salicin was used as an IS deviated substantially from 1.00 . When $\left[d_{4}\right]$-arbutin was employed as an SIL-IS, the \% ME values of arbutin and $\left[d_{4}\right]$-arbutin were in the ranges of $52.82-89.73$ and $60.73-78.1 \%$, respectively (Table $\mathrm{S} 1$ ), thereby indicating the similar ionisation suppression effects of arbutin and $\left[d_{4}\right]$-arbutin. Moreover, the matrix effect correction factors when $\left[d_{4}\right]$ arbutin was used as an IS were closer to 1.00 than the ones when salicin was used as a standard. Therefore, the problem of matrix effects in arbutin assays using LC-MS can be overcome by applying the stable isotope dilution approach.

\section{Method validation using LC-MS}

The calibration curve was determined using LC-MS with arbutin as a standard $(0.02-5.00 \mu \mathrm{g} / \mathrm{mL})$ and $\left[d_{4}\right]$-arbutin or salicin as an IS $(1.00 \mu \mathrm{g} / \mathrm{mL})$. The regression equation for $\left[d_{4}\right]$-arbutin was $y=0.8990 x+0.0016$, while for salicin it was found to be $y=0.1669 x+0.0378$. Furthermore, the correlation coefficients $\left(r^{2}\right)$ for the $\left[d_{4}\right]$-arbutin and salicin methods were measured to be 1.000 and 0.986 , respectively. The LOD and LOQ for the $\left[d_{4}\right]$-arbutin assay were calculated to be 0.03 and $0.10 \mu \mathrm{g} / \mathrm{mL}$, respectively, while for the salicin assay these values were 0.33 and $0.99 \mu \mathrm{g} / \mathrm{mL}$, respectively. Measurements on the precision (RSD\%) and accuracy (recovery \%) of the $\left[d_{4}\right]$-arbutin and salicin assays were taken in triplets with three different concentrations $(0.63,1.25$, and $5.00 \mu \mathrm{g} / \mathrm{mL})$ of arbutin standard within 1 day (Table 1). The precision of both the $\left[d_{4}\right]$-arbutin and salicin assays was $\leq 2.88 \%$, which is within the acceptable range stated in the AOAC guidelines (for $10 \mu \mathrm{g} / \mathrm{mL}: \leq 7.3 \%$, for $1 \mu \mathrm{g} / \mathrm{mL}: \leq 11.0 \%$ ). While the accuracy of the $\left[d_{4}\right]$-arbutin assay $(97.47-98.52 \%)$ was also within the acceptable range stated in the AOAC guidelines $(10 \mu \mathrm{g} / \mathrm{mL} ; 80-110 \%, 1 \mu \mathrm{g} / \mathrm{mL} ; 80-110 \%)$, the accuracy of the salicin assay (67.93-130.10\%) exceeded the reference values.

To assess the precision and accuracy of the method, blueberry leaf samples were prepared by spiking arbutin as a standard with two different concentrations $(0.10$ and $0.20 \mu \mathrm{g} / \mathrm{mL}$ ). Intra-day and inter-day variations of the arbutin analysis were performed both via LC-MS and GCMS using $\left[d_{4}\right]$-arbutin and salicin as ISs (Table 2). When the LC-MS method was applied, the precision values for $\left[d_{4}\right]$-arbutin and salicin were $\leq 2.8 \%$ in both the intra-day and inter-day variations, which is within the acceptable range stated in the AOAC guidelines (for $1 \mu \mathrm{g} / \mathrm{mL}$ : $\leq 11.0 \%)$. While the accuracy of $\left[d_{4}\right]$-arbutin (102.47-109.72\%) was within the acceptable ranges (for $1 \mu \mathrm{g} / \mathrm{mL}: \quad 80-110 \%)$, the accuracy of salicin (103.38-121.88\%) once again exceeded these values. The poor recovery $\%$ exhibited when salicin was used as an IS

Table 1 Precision (RSD, \%) and accuracy (recovery, \%) calculations from the calibration standards of arbutin obtained by LC-MS using salicin and $\left[d_{4}\right]$-arbutin as internal standard $(n=3)$

\begin{tabular}{llllll}
\hline Concentration $(\mu \mathrm{g} / \mathrm{mL})$ & \multicolumn{2}{l}{ Salicin } & & {$\left[d_{4}\right]$-arbutin } & \\
\cline { 2 - 3 } & Precision $(\mathrm{RSD}, \%)$ & Accuracy (recovery, \%) & & Precision (RSD, \%) & Accuracy (recovery, \%) \\
\hline 5.00 & 1.71 & $94.26 \pm 1.61$ & 1.80 & $98.24 \pm 1.77$ \\
1.25 & 2.88 & $122.32 \pm 3.52$ & 2.50 & $97.47 \pm 2.44$ \\
0.63 & 1.03 & $130.10 \pm 1.33$ & 0.12 & $98.52 \pm 0.12$ \\
\hline
\end{tabular}

$R S D$ relative standard deviation 


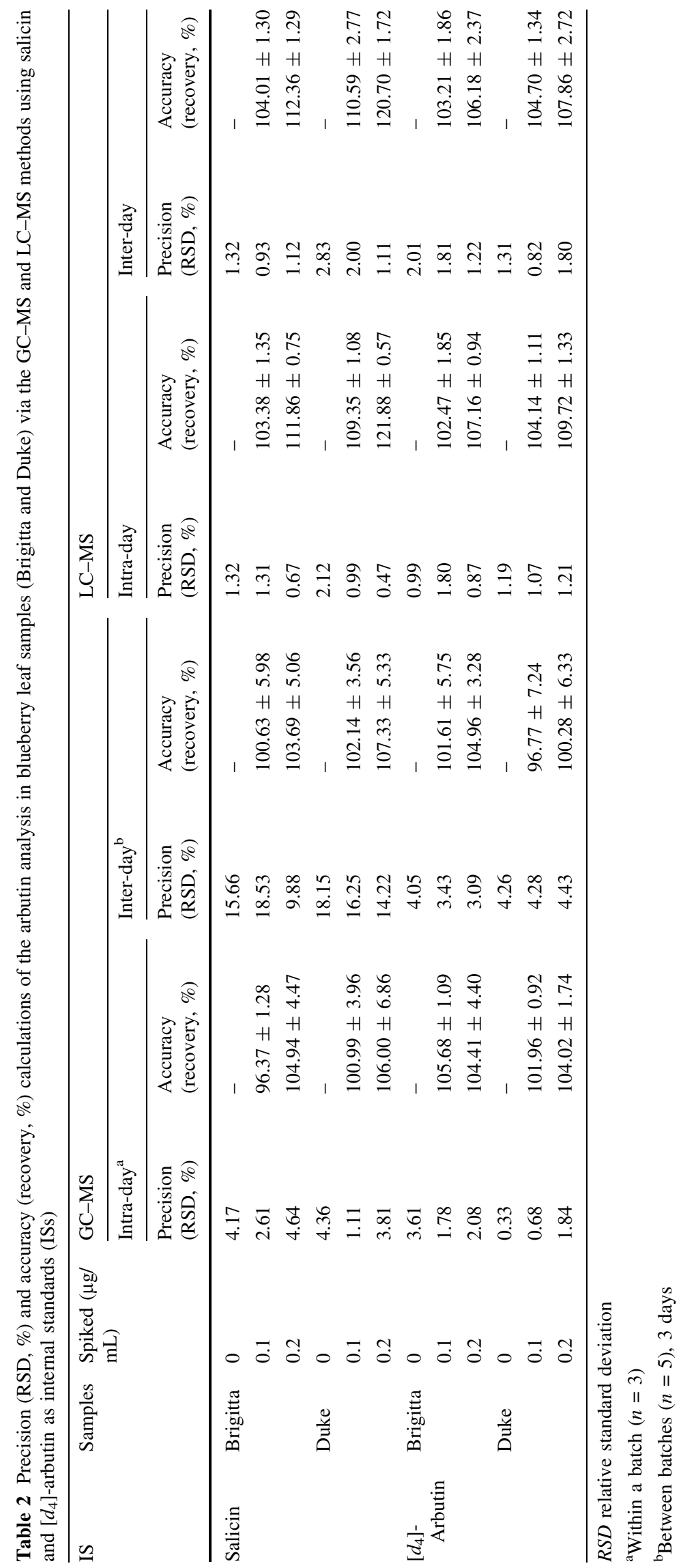


was caused by the ionisation suppression of the sample matrix effect, thereby indicating the lack of control over the matrix effect. Therefore, we developed and validated a method using $\left[d_{4}\right]$-arbutin as an IS which can control the matrix effect and have good precision and accuracy. Although repeatability tests for the validation of the $\left[d_{4}\right]-$ arbutin method using both GC-MS and LC-MS showed higher precision and accuracy results than the salicin method, the GC-MS method was substantially more timeconsuming (30 $\mathrm{min})$ than the LC-MS approach due to the additional derivatisation step required.

Next, the developed LC-MS method based on $\left[d_{4}\right]$-arbutin as an IS was used to detect arbutin in Brigitta $(0.43 \pm 0.01 \mu \mathrm{g} / \mathrm{mg}$ of dry weight, DW $)$ and Duke $(0.40 \pm 0.01 \mu \mathrm{g} / \mathrm{mg}$ of DW) blueberry leaves, as well as strawberry leaves $(0.16 \pm 0.02 \mu \mathrm{g} / \mathrm{mg}$ of fresh weight, FW) and pear peels $(6.86 \pm 1.39 \mu \mathrm{g} / \mathrm{mg}$ of DW). The results from these measurements were in agreement with previous studies, which report the concentrations of arbutin in blueberry leaves and pear peels to be $0.35 \pm 0.01 \mu \mathrm{g} /$ $\mathrm{mg}$ of DW and in the range of $0.92-27.70 \mu \mathrm{g} / \mathrm{mg}$ of DW, respectively [17, 35, 36]. To the best of our knowledge, the concentration of arbutin in strawberry leaves (Fragaria spp.) has not been previously reported.

In summary, by considering the matrix effect values, it was found that arbutin and $\left[d_{4}\right]$-arbutin exhibited almost the same matrix effect by co-eluting together, while salicin displayed a different matrix effect. Therefore, we developed a method for determining arbutin in plants using $\left[d_{4}\right]$ arbutin as an IS in an LC-MS analysis, which proved to be precise (precision $\leq 2.5 \%$, RSD $\%$ ), accurate (accuracy 97.47-98.52\%, recovery \%), and sensitive (linearity, $\left.r^{2}=1.000\right)$. In addition, the overall process of ionisation suppression of the sample matrix effect showed good recoveries (102.47-109.72\%) and matrix effect correction factors, thereby indicating that the matrix effect was overcome. Even though both the LC-MS and GC-MS methods using $\left[d_{4}\right]$-arbutin as an IS showed good precision and accuracy, the GC-MS approach was more time-consuming (by about $30 \mathrm{~min}$ ) than the LC-MS one due to the additional derivatisation step during the sample preparation. All in all, the novel method presented herein using a stable isotope dilution approach is expected to obtain reliable results in determining and quantifying arbutin in many more sample matrices.

Acknowledgments This work was supported by Funds from the Incheon National University Research in 2015, Republic of Korea.

\section{References}

1. Chakraborty AK, Funasaka Y, Komoto M, Ichihashi M (1998) Effect of arbutin on melanogenic proteins in human melanocytes. Pigment Cell Res 11:206-212

2. Kazuhisa S, Takahisa N, Koji N, Kenji S, Takashi K (2004) Inhibitory effects of $\alpha$-arbutin on melanin synthesis in cultured human melanoma cells and a three-dimensional human skin model. Biol Pharm Bull 27:510-514

3. Lukas B, Schmiderer C, Mitteregger U, Novak J (2010) Arbutin in marjoram and oregano. Food Chem 121:185-190

4. Masse M, Duvallet V, Borremans M, Goeyens L (2001) Identification and quantitative analysis of kojic acid and arbutine in skin-whitening cosmetics. Int J Cosmet Sci 23:219-232

5. Thogchai W, Liawruangrath B (2013) Micellar liquid chromatographic determination of arbutin and hydroquinone in medicinal plant extracts and commercial cosmetic products. Int $\mathrm{J}$ Cosmet Sci 35:257-263

6. Glockl I, Blaschke G, Veit M (2001) Validated methods for direct determination of hydroquinone glucuronide and sulfate in human urine after oral intake of bearberry leaf extract by capillary zone electrophoresis. J Chromatogr B Analyt Technol Biomed Life Sci 761:261-266

7. Lin YH, Yang YH, Wu SM (2007) Experimental design and capillary electrophoresis for simultaneous analysis of arbutin, kojic acid and hydroquinone in cosmetics. J Pharm Biomed Anal 44:279-282

8. Bulduk I, Sahin MD, Sanli S (2016) Arbutin analysis in leaves, fruit and branches of Pyrus anatolica, method optimization. Eurasian J Anal Chem 11:233-244

9. Escarpa A, Gonzalez M (2000) Evaluation of high-performance liquid chromatography for determination of phenolic compounds in pear horticultural cultivars. Chromatographia 51:37-43

10. Parejo I, Viladomat F, Bastida J, Codina C (2001) A single extraction step in the quantitative analysis of arbutin in bearberry (Arctostaphylos uva-ursi) leaves by high-performance liquid chromatography. Phytochem Anal 12:336-339

11. Cui T, Nakamura K, Ma L, Li J, Kayahara H (2005) Analyses of arbutin and chlorogenic acid, the major phenolic constituents in oriental pear. J Agric Food Chem 53:3882-3887

12. Inbaraj BS, Lu H, Kao T, Chen B (2010) Simultaneous determination of phenolic acids and flavonoids in Lycium barbarum Linnaeus by HPLC-DAD-ESI-MS. J Pharm Biomed Anal 51:549-556

13. Chisvert A, Sisternes J, Balaguer Á, Salvador A (2010) A gas chromatography-mass spectrometric method to determine skinwhitening agents in cosmetic products. Talanta 81:530-536

14. Lamien-Meda A, Lukas B, Schmiderer C, Franz C, Novak J (2009) Validation of a quantitative assay of arbutin using gas chromatography in Origanum majorana and Arctostaphylos uvaursi extracts. Phytochem Anal 20:416-420

15. Jurica K, Karačonji IB, Šegan S, Opsenica DM, Kremer D (2015) Quantitative analysis of arbutin and hydroquinone in strawberry tree (Arbutus unedo L., Ericaceae) leaves by gas chromatography-mass spectrometry. Arh Hig Rada Toksikol 66:197-202

16. Halket JM, Waterman D, Przyborowska AM, Patel RK, Fraser PD, Bramley PM (2004) Chemical derivatization and mass spectral libraries in metabolic profiling by GC/MS and LC/MS/ MS. J Exp Bot 56:219-243

17. Lee KB, Choi J, Ahn SK, Na JK, Shrestha KK, Nguon S, Kim JK (2018) Quantification of arbutin in plant extracts by stable isotope dilution gas chromatography-mass spectrometry. Chromatographia 81:533-538

18. Matuszewski BK, Constanzer ML, Chavez-Eng CM (2003) Strategies for the assessment of matrix effect in quantitative 
bioanalytical methods based on HPLC-MS/MS. Anal Chem 75:3019-3030

19. Taylor PJ (2005) Matrix effects: the Achilles heel of quantitative high-performance liquid chromatography-electrospray-tandem mass spectrometry. Clin Biochem 38:328-334

20. Van Eeckhaut A, Lanckmans K, Sarre S, Smolders I, Michotte Y (2009) Validation of bioanalytical LC-MS/MS assays: evaluation of matrix effects. J Chromatogr B Analyt Technol Biomed Life Sci 877:2198-2207

21. Silvestro L, Tarcomnicu I, Savu SR (2013) Matrix effects in mass spectrometry combined with separation methods-comparison HPLC, GC and discussion on methods to control these effects. In: Coelho AV, Franco CF (eds) Tandem mass spectrometrymolecular characterization. InTech Publisher, Rijeka, pp 3-37

22. Bonfiglio R, King RC, Olah TV, Merkle K (1999) The effects of sample preparation methods on the variability of the electrospray ionization response for model drug compounds. Rapid Commun Mass Spectrom 13:1175-1185

23. Zrostllkova J, Hajšlová J, Poustka J, Begany P (2002) Alternative calibration approaches to compensate the effect of co-extracted matrix components in liquid chromatography-electrospray ionisation tandem mass spectrometry analysis of pesticide residues in plant materials. J Chromatogr A 973:13-26

24. Alder L, Lüderitz S, Lindtner K, Stan HJ (2004) The ECHO technique - the more effective way of data evaluation in liquid chromatography-tandem mass spectrometry analysis. J Chromatogr A 1058:67-79

25. Wieling J (2002) LC-MS-MS experiences with internal standards. Chromatographia 55:S107-S113

26. Stokvis E, Rosing H, Beijnen JH (2005) Stable isotopically labeled internal standards in quantitative bioanalysis using liquid chromatography/mass spectrometry: necessity or not? Rapid Commun Mass Spectrom 19:401-407

27. Van De Steene JC, Lambert WE (2008) Comparison of matrix effects in HPLC-MS/MS and UPLC-MS/MS analysis of nine basic pharmaceuticals in surface waters. J Am Soc Mass Spectrom 19:713-718

28. Lang R, Yagar EF, Eggers R, Hofmann T (2008) Quantitative investigation of trigonelline, nicotinic acid, and nicotinamide in foods, urine, and plasma by means of LC-MS/MS and stable isotope dilution analysis. J Agric Food Chem 56:11114-11121

29. Park YJ, Park SU, Ha SH, Lim SH, Kim JK (2018) Improved quantification of $\gamma$-aminobutyric acid in rice using stable isotope dilution gas chromatography-mass spectrometry. Food Chem 266:375-380

30. Bruce SJ, Guy PA, Rezzi S, Ross AB (2010) Quantitative measurement of betaine and free choline in plasma, cereals and cereal products by isotope dilution LC-MS/MS. J Agric Food Chem 58:2055-2061

31. Paez V, Barrett WB, Deng X, Diaz-Amigo C, Fiedler K, Fuerer C, Hostetler GL, Johnson P, Joseph G, Konings EJM, Lacorn M, Lawry J, Liu H, Marceau E, Mastovska K, Monteroso L, Pan SJ, Parker C, Phillips MM, Popping B, Radcliffe S, Rimmer CA, Roder M, Schreiber A, Sealey-Voyksner J, Shippar J, Siantar DP, Sullivan DM, Sundgaard J, Szpylka J, Turner J, Wirthwine B, Wubben JL, Yadlapalli S, Yang J, Yeung JM, Zweigenbaum J, Coates SG (2016) AOAC SMPR(®) 2016.002. J AOAC 99:1122-1124

32. Bergeron A, Garofolo F (2013) Importance of matrix effects in LC-MS/MS bioanalysis. Bioanalysis 5:2331-2332

33. Kittipongpatana N, Chaiwan A, Pusod U, Kittipongpatana OS (2007) High-performance liquid chromatographic method for separation and quantitative analysis of arbutin in plant tissue cultures. CMU J Nat Sci 6:65-74

34. Mostafa SE, Karam NS, Shibli RA, Alali FQ (2010) Micropropagation and production of arbutin in oriental strawberry tree (Arbutus andrachne L.). Plant Cell, Tissue Organ Cult 103:111-121

35. Sasaki C, Yoshida Y, Asada C, Nakamura Y (2016) Total utilization of Japanese pear tree prunings: extraction of arbutin and production of bioethanol. J Mater Cycles Waste Manag 18:385-392

36. Kim J, Kim JI, Lee CW (2016) Development and validation of a modified QuEChERS method coupled with LC-MS/MS to determine arbutin in pear peels. Food Sci Biotechnol 25:987-992 